Review

\title{
Review of the Progress in Corneal Neovascularization Animal Models
}

\author{
${ }^{1,2}$ Shuci Tian, ${ }^{1}$ Shurong Wang, ${ }^{1}$ Yuxi He, ${ }^{1}$ Xin Liu, ${ }^{1}$ Ying Li, ${ }^{1}$ Guanfang Su and ${ }^{1}$ Yan Zhang \\ ${ }^{1}$ Department of Ophthalmology, The Second Hospital of JiLin University, Changchun 130041, China \\ ${ }^{2}$ Norman Bethune Health Science Center of JiLin University, Changchun 130021, China
}

\author{
Article history \\ Received: 21-05-2015 \\ Revised: 06-08-2015 \\ Accepted: 08-08-2015 \\ Corresponding Author: \\ Yan Zhang \\ Department of Ophthalmology, \\ the Second Hospital of JiLin \\ University, Changchun 130041 \\ China \\ Email: zhangy66@jlu.edu.cn
}

\begin{abstract}
Corneal Neovascularization (CNV), the extensive growth of blood vessels from conjunctiva into the cornea. It is a sight-threatening condition that can decreases eyesight and even leads to blindness. The pathologic growth of blood vessels impairs light transmission, promotes scar formation and causes inflammations, which harm visual acuity. Abnormal angiogenesis plays an important role in the process of CNV, which may result from corneal wound healing. Neovascular eye disease is one of the most common eye diseases in clinical admissions. CNV blinds approximately 7 million people worldwide. Continued mechanistic studies are a key to the prevention and treatment of CNV. Research in $\mathrm{CNV}$ animal models is essential in eye diseases. Several main methods of $\mathrm{CNV}$ models preparation are summarized in this review.
\end{abstract}

Keywords: Corneal, Vascularization, Model, Preparation

\section{Introduction}

Vasculogenesis is a natural process associated with growth, reproduction and the repair of damaged tissue. The influence of the new blood vessels depends on the location and the time of the vasculogenesis. Under physiological conditions, an extensive capillary network exists in the corneal limbus. Trauma and inflammation cause new blood vessels to invade the cornea, resulting in pathological CNV. Factors involved in the pathological process are listed as follows (Wang and Gao, 2010; Chen and Xu, 2001): (1) Vascular Factors: Vascular factors consist of inhibitory factors such as matrixmetalloproteins and stimulatory factors such as Vascular Endothelial Growth Factor (VEGF). The angiogenesis process is tightly regulated by numerous stimulatory and inhibitory factors. In the normal physiological state, the inhibitory factors are dominant. Pro- and anti-angiogenic factors reach a certain balance to maintain a stable state in the cornea. When the balance is disrupted, pro-angiogenic factors such as VEGF or basic Fibroblast Growth Factor (bFGF) trigger proliferation and migration of vascular endothelial cells into the surrounding tissues to form new blood vessels (McNatt et al., 1999). (2) Cornea Edema: Cornea tissue become loose: The spacing between the corneal layers widens and the blood vessels are able to invade. (3) Inflammatory Reaction:
Neutrophils' infiltration results in the formation of cell debris. They usually can be detected around the new blood vessels which will also prompt neovascularization (Azar, 2006). (4) Hypoxia: Hypoxia can stimulate tissue to produce angiogenesis factors to trigger angiogenesisis. The factors mentioned above have been confirmed by a series of experiments. (5) Corneal Nerve: New research finds that sensory nerves inhibit formation of neovessels and neovessels inhibit sensory nerves in the cornea. Nerve damage may play an important role in the process of corneal neovascularization (Ferrari et al., 2013). This viewpoint is newly presented, the specific relation between corneal nerves and corneal blood vessels remains futher disscuss.

The animal model of corneal neovascularization is prepared by surgery, physical or chemical methods to artificially induce the hyperplasia of neovascularization, which is similar to the human pathological process. CNV models should possess the following characteristics:

- The model is simple and stable

- The process is simple and easy

- The formation of the new blood vessels are regular

- The model can be prepared in large quantities

- The experimental conditions are simple

- There is little difference between individuals

- Other conditions are easy to control 
Table 1. This is a table covering the method mentioned in the review

\begin{tabular}{|c|c|c|c|}
\hline \multirow{3}{*}{$\frac{\mathrm{CNV} \text { models }}{\text { Inflammatory CNV model }}$} & \multicolumn{3}{|l|}{ Categories } \\
\hline & Physical method & & Chemical method \\
\hline & Suture induced CNV model & Thermal burn method & Chemical burn method \\
\hline CNV model induced by & Inducers & & \\
\hline sustained-released inducers & VEGF & bFGF & Endotoxin \\
\hline Immunologic CNV model & Xenogenic corneal transplantation & Allogeneic corneal transplantation & Injection of Bovine Albumin (BA) \\
\hline Other CNV models & $\begin{array}{l}\text { There are some models that are not } \\
\text { commonly used, such as mechanical } \\
\text { curettage of rabbit corneal } \\
\text { epithelium, tumor tissue implantation in } \\
\text { corneal stromal, removal of the } \\
\text { corneal and limbal epithelium. }\end{array}$ & & \\
\hline
\end{tabular}

All animal experiments should be accordant with the principles of the Association for Research in Vision and Ophthalmology (ARVO) Statement for the Use of Animals (Conners et al., 1995). The CNV model preparations are introduced in following several aspects: Model methods, results, principles, advantages and disadvantages.

In the following sections, we outline the current animal models and comment on the pros and cons of each. The following methods are all from literature. Table 1 summarizes the methods mentioned in this review.

\section{Inflammatory CNV Model}

\section{Inflammatory CNV Model Induced by Physical Methods}

\section{Suture Induced CNV Method}

Method (Park et al., 2015): Rabbits are anesthetized by intramuscular injection of $0.2 \mathrm{mg} \mathrm{kg}^{-1}$ of Zoletil 50 and $30 \mathrm{mg} \mathrm{kg}^{-1}$ of ketamine hydrochloride. After topical anesthesia of proparacaine, a corneal suture is placed at mid-stromal depth $1.0 \mathrm{~mm}$ near the limbus with a 3.0mm-wide black suture. Topical antibiotics are applied twice a day to prevent infection.

Results (Park et al., 2015): Normally, the new blood vessels appear in 3-8 days after the surgery. The vessels achieve a growth peak in 14 to 18 days. New blood vessels grow along the stitching direction and appear in both sides of the suture localized.

Principle: The suture in the matrix causes the local edema of the cornea and inflammatory cells invade the tissue (Azar, 2006). Cheng et al. (2014) found that the corneal suture caused the damage of the epithelial cells and basal cells. On the one hand, the suture causes corneal microenvironment hypoxia which triggers the expression of VEGF. Infiltration of inflammatory cells promotes angiogenesis factors which stimulate the high expression of VEGF.

Evaluations: Compared with the other methods mentioned in this review, the operation process is simple and this method is timesaving. The formation of the new blood vessels are relatively regular; the postoperative inflammation is easy to control. In addition, this is a physical method that could avoid the influence of chemical reagents (Tang et al., 2004). However, the result can be influenced by the skills of the surgeon. A deep suture can lead to corneal perforation. Whereas if a suture is too shallow, the suture can be easily scratched off by animals (Tang et al., 2004).

\section{Thermal Burn Method}

At present, there are few research reports about secondary angiogenesis after corneal thermal burn which restricts the basic study of the new blood vessels after thermal burn. In contrast, a constant-temperature burn method is widely used for corneal thermal burn.

Method (Jia et al., 2014): Set the temperature of the heater according to research needs. Anesthetize the rabbit by intramuscular injection, drip oxybuprocaine hydrochloride eye drops for the corneal surface anesthesia. Open the eyelid under the microscope, wipe the liquid to dry the cornea with a cotton swab, press the probe at central cornea and burn for $5 \mathrm{~s}$ and finally, wash the cornea with saline. The antibiotic ointments are applied to the corneal surface to prevent infection.

Results (Jia et al., 2014): New blood vessels appeared in the 4th day. The vessels reached the growth peak in the 14th day.

Principle: The neovascularization is on account of the secondary inflammatory reaction. A classical viewpoint is that the prostaglandin synthesis increases regionally after the thermal damage, the prostaglandin actives vascular growth factors also inhibits angiogenesis inhibitors steadily (Cooper et al., 1980).

Evaluations: Compared with other methods mentioned in this review, the operation procedure is simple and the experiment tools are common. However, this method can easily lead to corneal perforation and leukoma. There are many factors influencing the results of model preparation: Indoor temperature, humidity, tear and operation proficiency will influence the heating temperature (Jia et al., 2014). The repeatability of model preparation is poor.

\section{Chemical Burn Induced CNV Method}

Chemical Burns Method: In this method, alkaline chemicals are applied to burn the cornea and induce new 
blood vessels. Commonly used chemicals include sodium hydroxide, silver nitrate and potassium nitrate.

Method (Kuerten et al., 2015): Rabbits are placed under general anesthesia by intramuscular injection of ketamine/xylazine ( 35 and $5 \mathrm{mg} \mathrm{kg}^{-1}$ respectively). The cornea is anesthetized by topical application of $0.5 \%$ proparacaine hydrochloride. A $5 \mathrm{~mm}$ diameter filter paper disc soaked in $1.0 \mathrm{M} \mathrm{NaOH}$ for $1 \mathrm{~min}$ is placed on the corneal surface, 1-3 mm away from the limbus. The disc is removed immediately and the eye is rinsed thoroughly with $10 \mathrm{~mL}$ saline after $2 \mathrm{~min}$. Antibiotic ointments are applied to the corneal surface to prevent infection.

Moreover, There's a new found that the combined application of bFGF and alkali burn to induce new blood vessels can increase and maintain the growth level of blood vessels. This method also reduces the strength of alkali burn which effectively avoids complications such as dissolution and extrusion and results in improves success rate (Guo et al., 2014).

Results (Kuerten et al., 2015): After 3 days from the alkali burn, the vascular network in the limbus sclera expanded with new blood vessels growing rapidly on the cornea and extending deeply into the cornea limbus. The vessels reached a growth peak in 7 days.

Principle: When a basic solution touches the cornea, alkali enters into cells and destroys the structure of the cells. The immunomechanism of neovascularization is widely accepted in recent years (Xing, 2010): Denatured proteins become antigens and cause an immune response. In addition, the corneal trauma stimulates chemokines such as Platelet Activating Factor (PAF) to induce the PMN (polymorphonuclear) cells to enter the cornea. PMN cells stimulate the expression of lymphokines such as IL-1, TNF- $\alpha$ to trigger as well as maintain the corneal inflamation. The basic solution also takes into effect by triggering the inflammatory cells to express VEGF to induce CNV.

Evaluations: It is the simplest method and the cost is low (Birsner et al., 2014). It is one of the most frequently used method in corneal neovascularization animal model preparation. In addition, the animal model of alkaline burn reflects the pathological condition of the ocular chemical burn disease very well.

\section{CNV Model Induced by Sustained-Released Inducers}

Compared to other methods mentioned in this review, this method is particularly useful when used to identify stimulators of angiogenesis. However, the operation process is complex and the surgical injury can also cause CNV which affects the experimental results.

Basic method: For surgical implantation and gradual release of the factor, the polymers should be brought in a semisolid state. Consequently, the polymers are manufactured into pills. Then cut the cornea for a tunnel, embed the pills containing specific growth factors in the cornea layer to trigger angiogenesis. New blood vessels can be measured and quantified (Mao et al., 2014). Here are some common inducers: VEGF, bFGF, interleukin-1 (IL-1), endotoxin and silicon dioxide.

\section{CNV Induced by Sustained-Release Polymer Pills Containing VEGF}

Method (Coman et al., 2010): Preparation of sustained-release VEGF polymers: Isoform VEGF165 (with 165 amino acids) each containing $5 \mu \mathrm{g}$ VEGF can be used with any buffer solution was prepared. For surgical implantation and gradual release of the factor, VEGF should be brought in a semisolid state. Each pill contained about 100 or 200 ng VEGF accompanied with sucralfate.

The VEGF pills are embedded in the corneal pocket under sterile conditions.

Results (Coman et al., 2010): Moderate corneal edema is detected $24 \mathrm{~h}$ after the intervention. In the eyes implanted with $200 \mathrm{ng}$ VEGF, the normal limbic vessels dilated in the first two days. On day 3 , the limbic vessels advanced to the cornea. Day 4-5, the new blood vessels extended to the pellet. On day 6, these new blood vessels invaded the pellet. Starting with day 7 , the angionenesis reaction became stable. The eyes implanted with pellets containing a smaller dosage of VEGF (100 ng) showed significantly lower angionenesis reactions. Moreover, starting with day 7, a slight stromal edema and tissue hemorrhage happened in the eyes implanted with $200 \mathrm{ng}$ VEGF, which were absent at the lower dose of VEGF (100 ng).

Principle: VEGF, a polypeptide growth factor, has important roles in blood vessels. It's one of the most important factors in the regulation of $\mathrm{CNV}$ formation. It also act as the control center of new blood vessels proliferation (Zhao et al., 2007). VEGF acts on vascular endothelial cells directly and combines with the specific receptor (VEGFR-I and VEGFR-II) on vascular endothelial cells to activate the tyrosine kinase and downstream signaling cascades to promote migration and proliferation of the endothelial cells and angiogenesis (Zhao et al., 2007).

Evaluations: VEGF is derectly stimulartion factor. It can stimulate the new blood vessels directly which benefits the therapeutic evaluation of agiogenesis inhibitors.

\section{CNV Induced by Sustained-Release Polymer Pills Containing $b F G F$}

Method (Morbidelli and Ziche, 2004): First, the bFGF slow-released pills are prepared. The medical gelatin sponge is cut into $1 \mathrm{~mm} * 1 \mathrm{~mm} * 0.5 \mathrm{~mm}$ pieces 
with a microscopic shear under the microscope, the pieces are immersed in $4^{\circ} \mathrm{C}$ PBS buffer for one night and dried with sterile filter papers. $1 \mu \mathrm{L}$ bFGF (500 ng $\left.\mu \mathrm{L}^{-1} \mathrm{PBS}\right)$ is dropped on each piece and the pieces are settled in the clean bench for $48 \mathrm{~h}$. Wrap up the pills with $2 \%$ agarose solution and the pills are stored in the clean hood for $48 \mathrm{~h}$.

The bFGF pills are embedded in the corneal pocket under sterile conditions.

Results: The new blood vessels develop from the corneal limbus in average $4.00 \pm 0.83$ days. In addition, the blood vessels extended in the direction of the implantation materials and gradually wrap the implantation materials in bundles (Morbidelli and Ziche, 2004).

Principle: bFGF promotes the differentiation, proliferation, migration and chemotaxis of endothelial cells. bFGF increases capillaries production by stimulating mitosis. Additional hormone-like activity influences corneal diaphaneity (Hecquet et al., 1990; Bremnes et al., 2006). bFGF promotes the secretion of collagen fiber. bFGF induces endothelial cells to produce a large number of urokinase type fibrinolytic enzyme activators (urine lpasminogen activator, u-PA) which work in early angiogenesis to dissolve collagenase enzyme. Collagenase will dissolve the collagen of the vessels margin and lead the endothelial cells into the three-dimensional collagen matrix to form new blood vessels sprouts (Qun-Xiu et al., 2010). Moreover, Yang et al. (2000) found that bFGF's effect on neovascularization is associated with a positive linear relationship on dose (Yang et al., 2000).

Evaluations: bFGF can stimulate the new blood vessels directly which benefits the therapeutic evaluation of agiogenesis inhibitors. The model's complex operation and overmuch influence factors make it hard to control the growth.

\section{CNV Model Induced by Sustained-Release Endotoxin Polymer}

This method is modified to produce $\mathrm{CNV}$ by implanting into the cornea a sustained-released polymer containing endotoxin (Escherichia coli lipopolysaccharide). Endotoxin is lipopolysaccharides, a component of cell walls of gram-negative bacteria. It's a reliable model for investigating angiogenesis inhibitors.

Method (Li et al., 1991; Jin et al., 1998): Preparation of sustained-released endotoxin polymers: Polymers containing endotoxin (E. coli lipopolysaccharide; Sigma, St. Louis, MO) loads of 15,30 and $40 \%$ are produced. High-grade absolute ethanol was used to wash the Ethylene vinyl acetate copolymer (Elvax; Dupont, Wil-mington, DE) beads.
The polymer was dissolved in methylene chloriden and diluted to a concentration of $10 \%$. An eight-well Teflon mold is prechilled on dry ice, with its working surface covered with a glass plate to prevent condensation in the wells. The endotoxin-Elvax suspension is delivered through a sterile glass pipette and allowed to polymerize for $10 \mathrm{~min}$. The $10 \%$ Elvax and vortex are mixed with lyophilized endotoxin. The endotoxinElvas solid are stored at $-20^{\circ}$ for $24 \mathrm{~h}$ to complete polymerization and then desiccated for $48 \mathrm{~h}$ to evaporate residual solvent. The polymer pills are cut into approximately $1 \mathrm{mg}$ pills. Polymer pills should be sterilized by ultraviolet irradiation before implantation.

The endotoxin pills are embedded in the corneal pocket under sterile conditions.

Results (Li et al., 1991; Zhang and $\mathrm{Hu}, 2006$ ): The results reveal that the eyes implanted with $15 \%$ endotoxin-polymer produce negligible corneal edema that maintained only 3-4 days after the operation. The eyes implanted with higher levels of endotoxin (30 and $40 \%$ ) induce a substantial neovascular response accompanied by severe corneal edema occurring in 24 $\mathrm{h}$ after implantation. In the eyes implanted with $15 \%$ endotoxin-polymer, newly formed vessels grow from the limbus toward the pocket at the 16th day after the operation.

New blood vessels induced by endotoxin have a dependence on local endotoxin. Models can be induced to adapt the research needs in different concentration of endotoxin. However, high concentration of endotoxin can cause corneal matrix turbidity and new blood vessels tend to coalesce which influences the quantitative analysis of the vessels. If the concentration is too low, few vessel are made that can reach the requirement of the model (Li et al., 1991).

Evaluations (Zhang and $\mathrm{Hu}, 2006)$ : The operation procedure is complex. Surgical injury can also cause CNV which affects the experimental results. The scope of the new blood vessels in this model accounts for only 10$20 \%$ of corneal circumference which is convenient for researchers to measure them dynamically. Moreover, the severe, moderate, mild CNV models can be induced with different concentration of the endotoxin to adapt different research needs.

\section{Immunologic CNV Model}

The Immunologic CNV method includes xenogenic corneal transplantation, allogeneic corneal transplantation and injection of Bovine Albumin (BA), which are used to investigate the regulation mechanism of the rejection reaction and to evaluate treatment methods (Damms et al., 1997). 


\section{Allogeneic Corneal Transplantation to Induce CNV}

Method (Damms et al., 1997): The rabbits are divided into two groups randomly, one group being donors and another being receptors. The donors are operated on to obtain the cornea as corneal graft. Anesthetize the recipients, a plant bed is manufactured with a $7.25 \mathrm{~mm}$ trephine, the donor cornea and the plant bed are sutured symmetrically together, using 1216 needles in total. Erythromycin ointment is applied to the corneal surface to prevent infection.

Results (Damms et al., 1997): A certain degree of corneal edema appears 3-4 days after the operation, the edema at the junction area of the donor graft and plant bed is more evident. Capillary sprouts invade the limbus 7 days after the operation, new blood vessels grow exuberantly to the edge of the wound accompanied with diffuse edema in donor graft. About 14 days later, the edema in the donor graft is severe. New blood vessels extend to the central cornea. Rejection reaction occurs and the average survival time of the donor graft is $10.0 \pm 1.5$ days.

Principle: The HLA antigen system is active in the cornea and has diversity. HLA is a kind of allogeneic antigen with highly polymorphic, HLA class I antigen distributes in all nucleated cells. When allogeneic cornea come into body, the HLA class I antigen can be recognized by the receptor's immune system. Allogeneic cornea becomes antigens and combines with antibodies to form antigen-antibody complexes, activate complement system, thus causing an immune response. This leads to infiltration of lymphocytes and factors, inducing $\mathrm{CNV}$ formation.

Evaluations: The mechanism of the model is identical with the mechanism of human corneal transplantation rejection. It's the ideal model in researching the mechanism, etiology and treatment of the rejection after cornea transplantation.

\section{Other Animal CNV Models}

There are many factors that can lead to the proliferation of CNV. A variety of animal models have developed to study the pathogenesis of CNV disease. Apart from the CNV models above, there are additional modeling methods that are not commonly used, such as mechanical curettage of rabbit corneal epithelium, tumor tissue implantation in corneal stromal, removal of the corneal and limbal epithelium to induce rabbit CNV models (Amano et al., 1998). However, compared with the model methods above, these models have more obvious disadvantages, such as the response consistency is poor, the quantitative analysis is difficult and the operational process is complex (Regenfuss et al., 2008; Shi et al., 2010).

\section{Therapeutics of CNV}

New blood vessels block light and compromise visual acuity. Many researchers contribute themselves to find out the therapeutics of CNV. The animal models of corneal neovascularization are applied to therapeutic researches. There are many approaches to treat new blood vessels. Inhibitors of angiogenesis factors' expression have always been the hot issues in therapeutic of CNV.

Apart from the therapeutics mentioned above, there are some other methods in treatment of CNV diseases. For instance, surgical treatment such autologous corneal transplantationas has been applied to clinic. Gene therapy and laser therapy was confirmed therapeutic but need further studies. Some other medications such as some inhibitors which block the receptors of the angiogenesis factors and inhibitors which suppress the interactions between endothelial cells and extracellular matrix are also applied in preclinical experiments. Table 2 summarizes some significant inhibitors of angiogenesis factors' expression.

Table 2. This is a table covering some significant inhibitors of angiogenesis factors' expression

Inhibitors of angiogenesis factors'

expression (Sun and Zhang, 2006) Applications

Antibodies

Anti-VEGF, anti-bFGF and anti-interleukin 8(IL-8) have been widely employed in

experiments especially anti-VEGF: Bevacizumab (Avastin) and ranibizumab

(lucentis) have been applied to clinical therapy; Pegaptanib (Macugen),

Bevacizumab (Avastin), ranibizumab (lucentis) and VEGF

Trap-Eye/Aflibercept (eylea) have entered the III phase of clinical trial.

Suramin

Suramin is a kind of anti-tumor drugs. Its therapeutic effects on neovascular eye diseases are still in the phase of preclinical study.

Curcumin Curcumin suppress the NFאB pathway and the expression of VEGF, inflammatory cytokine and Matrix Metalloproteinases (MMPs) (Pradhan et al., 2015). Its therapeutic effects on neovascular eye diseases are still in the phase of preclinical study.

Other inhibitors There are many inhibitors which have effects on angiogenesis inhibiting: Linomide, tecogalan sodium, spironolactone and etc. 


\section{Conclusion}

The animal models of corneal neovascularization presented above have their own advantages and disadvantages. CNV models induced by suture and chemicals are widely used in corneal experiments. CNV models induced by sustained-released inducers are widely used in the study of the stimulators of angiogenesis. Immunologic $\mathrm{CNV}$ models are mainly applied to the study of transplantation rejection. The animal model of corneal neovascularization is the foundation of human neovascular eye disease research.

To date, there are many good modeling systems, designed to fulfill different requirement during the investigation of pathogenesis of CNV. It benefits from screening drug in pre-clinical state to treating human corneal disease in clinical settings.

\section{Acknowledgment}

The authors would like to thank the funding support from Science and Technology Department $(20130413025 \mathrm{GH})$ and Health Department of Jilin province, China (2013Q005).

\section{Author's Contributions}

Shuci Tian: Data collection and manuscript writing.

Shurong Wang: Conception and design, data collection and manuscript revision. Contributed equally to this study, share first authorship.

Xin Liu, Yuxi He and Ying Li: Data collection.

Yan Zhang: Conception, revision and final approval of the version.

\section{Ethics}

All authors read and approved the final version and are responsible for any ethical issue that may arise after the publication of this manuscript.

\section{References}

Amano, S., R. Rohan, M. Kuroki, M. Tolentino and A.P. Adamis, 1998. Requirement for vascular endothelial growth factor in wound- and inflammation-related corneal neovascularization. Invest. Ophthalmol. Vis. Sci., 39: 18-22. PMID: 9430540

Azar, D.T., 2006. Corneal angiogenic privilege: Angiogenic and antiangiogenic factors in corneal avascularity, vasculogenesis and wound healing (an American Ophthalmological Society thesis). Trans. Am. Ophthalmol. Soc., 104: 264-302.

Birsner, A.E., O. Benny and R.J. D'Amato, 2014. The corneal micropocket assay: A model of angiogenesis in the mouse eye. J. Vis. Exp.

DOI: $10.3791 / 51375$ (2014)
Bremnes, R.M., C. Camps and R. Sirera, 2006. Angiogenesis in non-small cell lung cancer: The prognostic impact of neoangiogenesis and the cytokines VEGF and bFGF in tumours and blood. Lung Cancer, 51: 143-158. PMID: 16360975

Chen, J.S. and J.T. Xu, 2001. Corneal neovascularization. Ophthalmol. CHN., 10: 137-143.

Cheng, G.H., W. Jiang and Y.Q. Zhu, 2014. Study on the inhibition of interleukin-1 recept or antagonist on the growth of corneal neovascularization and the expression of vascular endothelial growth factor. Aminoacids Biotic.

Coman, L., O.A. Coman, H. Păunescu, F. Drăghia and I. Fulga et al., 2010. VEGF-induced corneal neovascularisation in a rabbit experimental model. Rom. J. Morphol. Embryol., 51: 327-336. PMID: 20495752

Conners, M.S., R.A. Stoltz, S.C. Webb, J. Rosenberg and M.W. Dunn et al., 1995. A closed eye contact lens model of corneal inflammation. Part 1: Increased synthesis of cytochrome P450 arachidonic acid metabolites. Invest. Ophthalmol. Vis. Sci., 36: 828-840. PMID: 7706031

Cooper, C.A., M.V. Bergamini and I.H. Leopold, 1980. Use of flurbiprofen to inhibit corneal neovascularization. Arch. Ophthalmol., 98: 1102-1105.

DOI: 10.1001/archopht.1980.01020031092017

Damms, T., J.R. Ross and M.D. Duplessie, 1997. Intracorneal bovine albumin: An immunologic model of corneal angiogenesis. Graefe's Arch Clin. Exp. Ophthalmol., 235: 460-666. PMID: 9349952

Ferrari, G., A.R. Hajrasouliha, Z. Sadrai, H. Ueno and S.K. Chauhan et al., 2013. Nerves and neovessels inhibit each other in the cornea. Invest Ophthalmol. Vis., 54: 813-820. PMID: 23307967

Guo, L.Y., J.Y. Zhang and H. Sun, 2014. Effect of basic fibroblast growth factor on neovscularization in alkali burned rabbit corneas. Shandong Yiyao., 54: 7-9.

Hecquet, C., S. Morisset, G. Lorans, J. Plouet and M. Adolphe et al., 1990. Effects of acidic and basic fibroblast growth factors on the proliferation of rabbit corneal cells. Curr. Eye Res., 9: 429-433. DOI: $10.3109 / 02713689008999608$

Jia, Y., H. Jiang and Y.Q. Wang, 2014. Preliminary study of rabbit model with corneal neovascularization after thermal burn under the constant temperature. Guoji. Yanke. Zazhi., 14: 1193-119. DOI: 10.3980/j.issn.1672-5123.2014.07.04

Jin, L., Z.D. Cai and Y.F. Lou, 1998. An experimental study of corneal neovascularization model in the rabbit induced by the lyophilized endotoxin. Yanke Yanjiu, 16: 85-86. 
Kuerten, D., S. Johnen, N. Harmening, G. Souteyrand and P. Walter et al., 2015. Transplantation of PEDFtransfected pigment epithelial cells inhibits corneal neovascularization in a rabbit model. Gradfes Arch. Clin. Exp. Ophthalmol., 253: 1061-1069. PMID: 25690979

Li, W.W., G. Grayson, J. Folkman and P.A. D'Amore et al., 1991. Sustained-release endotoxin. A model for inducing corneal neovascularization. Invest. Ophthalmol. Vis. Sci., 32: 2906-2911. PMID: 1917394

Mao, Y.N., Y. Hu and S.P. Hou, 2014. Correlation between microRNA expression profiles and VEGF of corneal neovascularization in rats. J. Chongq. Med. Univ., 38: 1090-1094.

McNatt, L.G., L. Weimer, J. Yanni and A.F. Clark, 1999. Angiostatic activity of steroids in the chick embryo CAM and rabbit cornea models of neovascularization. J. Ocul. Pharmacol. Ther., 15: 413-423. DOI: 10.1089/jop.1999.15.413

Morbidelli, L. and M. Ziche, 2004. The rabbit corneal pocket assay for the study of angiogenesis. Cancer Treat Res., 117: 147-151. DOI: $10.1007 / 978-1-4419-8871-3 \_10$

Park, J.H., C.K. Joo and S.K. Chung, 2015. Comparative study of tacrolimus and bevacizumab on corneal neovascularization in rabbits. Cornea, 34: 449-455. DOI: 10.1097/ICO.0000000000000336

Pradhan, N., R. Guha, S. Chowdhury, S. Nandi and A. Konar et al., 2015. Curcumin nanoparticles inhibit corneal neovascularization. J. Mol. Med. (Berl).

Qun-Xiu, L., L. Xue-Zheng, Z. Ke-Jian and H. Yang, 2010. Differentiation of rat bone marrow mesenchymal stem cells into nerve-like cells through co-culture with retinal cells and application of basic fibroblast growth factor in vitro. Chinese J. Optometry Ophthalmol. Visual Sci., 12: 122-123. DOI: 10.3760/cma.j.issn.1674-845X.2010.02.011
Regenfuss, B., F. Bock, A. Parthasarathy and C. Cursiefen, 2008. Corneal (lymph)angiogenesis-from bedside to bench and back: A tribute to Judah Folkman. Lymphat Res. Biol., 6: 191-201. PMID: 19093792

Shi, W., J. Liu, M. Li, H. Gao and T. Wang, 2010. Expression of MMP, HPSE and FAP in stroma promoted corneal neovascularization induced by different etiological factors. Curr. Eye Res., 35: 967-977. PMID: 20958185

Sun, G.L. and M.C. Zhang, 2006. Therapeutic advance of corneal neovascularization. Rec. Adv. Ophthalmo., 26: 313-316.

Tang, W.Q., L. Liu and J. Li, 2004. Corneal neovascular model construction in rats induced by corneal stitch. Int. J. Ophthalmol., 4: 820-823.

Wang, J.H. and G.P. Gao, 2010. Corneal vascularization and treatment. J. Clin. Rehabilitative Tissue Eng. Res., 14: 6223-6225.

Xing, Z.M., 2010. Research advance in immunological mechanism after corneal alkali burns. Cornea Alkali Burn Immunol., 28: 796-800.

Yang, C.F., T. Yasukawa, H. Kimura, H. Miyamoto and Y. Honda et al., 2000. Experimental corneal neovascularization by basic fibroblast growth factor incorporated into gelatin hydrogel. Ophthalmic. Res., 329: 19-24. PMID: 10657751

Zhang, L. and Y.H. Hu, 2006. Development of corneal neovascularization model induce $d$ by new corneal pocket method in rabbit. Rec. Adv. Ophthalmol., 26: 354-356.

DOI: 10.6040/j.issn.1671-7554.0.2013.498

Zhao, W., S.Q. Ling and Z.G. Liu, 2007. Study on rat models of corneal neovasculariztion induced by micro-pocket assay. Shiyong Yishi Zazhi, 14: 3425-3427. 